\title{
Motivos para Prática de Atividade Física e Imagem Corporal em Frequentadores de Academia
}

\author{
Reasons for Physical Activity Practice and Body Image Among \\ Health Clubs Users
}

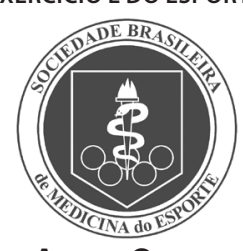

Artigo Original

Rogério César Fermino 1,2

Mariana Ramos Pezzini ${ }^{3}$

Rodrigo Siqueira Reis $1,2,3$

1. Grupo de Pesquisa em Atividade Física e Qualidade de Vida, Pontifícia Universidade Católica do Paraná GPAQ/PUCPR, Brasil.

2. Programa de Pós-Graduação em Educação Física, Universidade Federal do Paraná - UFPR, Brasil.

3. Curso de Educação Física,

Pontifícia Universidade Católica do

Paraná - PUCPR, Brasil.

\section{Endereço para correspondência:}

Rogério César Fermino

Pontifícia Universidade Católica do

Paraná - PUCPR

Centro de Ciências Biológicas e da

Saúde, CCBS - Curso de Educação

Física

Grupo de Pesquisa em Atividade

Física e Qualidade de Vida - GPAQ

Rua Imaculada Conceição, 1.155 -

Prado Velho

80215-901 - Curitiba, PR - Brasil

E-mail: rogeriofermino@hotmail.com

\section{RESUMO}

O objetivo deste estudo foi identificar os motivos para a prática de atividade física e analisar sua relação com a imagem corporal em adultos frequentadores de academia. A amostra foi selecionada de maneira intencional entre os indivíduos que frequentavam as atividades oferecidas pelo centro de esportes da PUCPR, sendo que 90 sujeitos (50\% de homens) completaram as etapas da pesquisa. Foram realizadas medidas antropométricas (massa corporal, estatura e dobras cutâneas) e aplicado um questionário contendo perguntas sobre motivos para a prática de atividade física e a escala de avaliação da figura corporal. A avaliação dos riscos relacionados com o sobrepeso/obesidade foi realizada com base no percentual de gordura (\%G) e índice de massa corporal (IMC). Os escores de motivos para a atividade física e da imagem corporal foram comparadas entre os sexos, categorias de \% G IMC através do teste $t$ de medidas independentes e correlação de Pearson. A regressão linear múltipla foi utilizada para analisar a associação entre as variáveis de estudo. Os maiores escores foram encontrados nas questões saúde e aptidão física. Para as mulheres e aqueles com \%G elevado, o motivo harmonia apresentou diferença significativa. Mulheres, indivíduos com \%G elevado e com excesso de peso apresentaram maior insatisfação com a imagem corporal. O motivo controle de peso e harmonia está associado ao \%G, IMC (exceto para harmonia) e insatisfação com a imagem corporal. As análises parciais demonstraram que o controle de peso não estava associado com a insatisfação corporal após ajuste para as variáveis controladoras em ambos os modelos e a variável harmonia apresentou associação apenas no primeiro modelo. Conclui-se que os motivos mais importantes foram saúde e aptidão física e que a insatisfação com a imagem corporal parece estar mais associada a aspectos relacionados com o bem-estar do que com a condição física.

Palavras-chave: motivação, exercício, autoimagem, tipo físico, índice de massa corporal.

\section{ABSTRACT}

The aim of this study was to identify the reasons for physical activity practice and its relationship with body image among health clubs users. The sample was intentionally selected among individuals who were engaged in exercising and sports at the Catholic University of Parana sports center and 90 subjects completed the study (50\% males). Anthropometric measurements (body mass, height and skinfolds) were taken as well as a questionnaire about reasons for physical activities and body figures evaluation was applied. The health risks related to overweight/obesity were determined based on relative body fat (\%F) and body mass index (BMI). The reason for physical activity and body image scores were compared according to gender, \%F and $\mathrm{BMI}$ categories through independent $\mathrm{t}$ test and with Pearson correlation. Multiple linear regression models were employed to analyze all variables associations. The highest scores were found in questions on health and physical fitness. Women and those with higher \%F'harmony'showed significant difference. Women and those with higher \%F or overweight showed more body image dissatisfaction. The reasons 'weight control' and 'harmony' were associated to \%F, BMI (except 'harmony') and dissatisfaction with body image. The partial analyses showed that weight control was not associated with body dissatisfaction after adjustment for control variables in both models and the variable harmony showed association only on the first model. In conclusion, the most important motives were health and physical fitness and dissatisfaction with body image seems to be more associated with aspects related to wellness than physical fitness.

Keywords: motivation, exercise, self-image, physical type, body mass index. 


\section{INTRODUÇÃO}

Imagem corporal é um conceito multidimensional que descreve amplamente as representações internas da estrutura corporal e aparência física, em relação a nós mesmos e aos outros ${ }^{(1,2)}$. O componente subjetivo se refere à satisfação de uma pessoa com seu tamanho corporal ou partes específicas ${ }^{(3)}$. Atualmente, alguns pesquisadores em ciências do esporte e exercício têm estado interessados em sua avaliação, pois se acredita que ela possa exercer um importante papel na seleção da atividade física e aderência continuada aos programas de exercício ${ }^{(2,4)}$.

$\mathrm{O}$ padrão de beleza valorizado na sociedade associado à magreza acaba por salientar os aspectos relacionados com a forma, desconsiderando a diversidade das constituições físicas que estão presentes na população e também aspectos de saúde ${ }^{(1,3,6)}$. Apesar de existirem valores de índice de massa corporal (IMC) e percentual de gordura (\%G) adequados para a saúde, acredita-se que o tipo físico possa ser determinado culturalmente ${ }^{(1,2,7)}$. Indivíduos obesos apresentam imagens corporais negativas, o que evidencia a composição corporal como um dos fatores capazes de influenciar tal percepção(4,8). Essa insatisfação tem sido um dos principais motivos que levam as pessoas a realizarem atividade física em busca de melhor aparência ${ }^{(9)}$, o que faz aumentar a procura de academias, clubes ou centros de saúde ${ }^{(2,10)}$.

De fato, os exercícios têm sido utilizados como um meio para reduzir o peso corporal de indivíduos insatisfeitos com a própria imagem ${ }^{(1,5,8-11)}$. Contudo, uma imagem corporal negativa pode, paradoxalmente, diminuir a motivação para a prática de atividades físicas ou aumentar o engajamento em programas de exercícios ${ }^{(4)}$. Hausenblas e Fallon ${ }^{(11)}$ verificaram que o exercício proporciona diminuição da depressão, meIhoria do bem-estar psicológico e que indivíduos ativos apresentam imagem corporal mais positiva, independente da idade e sexo. Entretanto, a relação com o exercício pode ser dependente de fatores como condição de saúde e composição corporal, por exemplo.

A prevalência de inatividade física é elevada em diversas capitais brasileiras ${ }^{(12,13)}$ em virtude de um baixo número de pessoas que praticam exercícios físicos ${ }^{(14)}$. Alguns estudos procuraram verificar os motivos para participação em programas de atividade física ${ }^{(15)} \mathrm{em}$ academias ${ }^{(10,16)}$ ou clínicas ${ }^{(17)}$. Os mais citados foram as questões estéticas, saúde e qualidade de vida ${ }^{(10,16)}$

Ainda que a insatisfação com a imagem corporal possa ser um importante aspecto para a procura de programas de atividade física como forma de controle do peso ${ }^{(1,4,8,9)}$, nem sempre esse fato reflete a real necessidade. De acordo com a revisão efetuada pelo autores, não foram encontrados estudos que tenham investigado essa abordagem. O objetivo deste estudo foi identificar os motivos para a prática de atividade física e analisar sua relação com a imagem corporal em indivíduos adultos frequentadores de academia.

\section{MÉTODOS}

\section{Amostra}

Foi realizado um estudo exploratório correlacional com adultos de ambos os sexos frequentadores dos programas de atividades físicas oferecidos pelo centro de esportes da Pontifícia Universidade Católica do Paraná (PUCPR). No período da realização da pesquisa a instituição possuía aproximadamente 300 participantes regularmente inscritos nas modalidades musculação, ergometria, ciclismo indoor e ginástica localizada. Os indivíduos que realizavam as atividades regularmente $(\geq$ três vezes por semana) a pelo menos seis meses foram convidados a participar do estudo e deveriam apresentar disponibilidade e interesse para tanto. A amostra foi selecionada de maneira intencional, sendo que 90 sujeitos completaram as etapas da pesquisa (preenchimento do questionário e avaliação antropométrica).

\section{Coleta de dados e instrumentos}

As informações foram coletadas com um formulário para o registro de dados antropométricos e um questionário composto pelo inventário de razões para o exercício, escala de avaliação da figura corporal, informações sociodemográficas e de atividade física. As avaliações foram realizadas individualmente, em ambiente apropriado, por um dos autores do estudo que recebeu treinamento prévio.

Foram coletados os dados antropométricos de massa corporal (MC, $\mathrm{kg}$ ), estatura (EST, $\mathrm{cm}$ ) e dobras cutâneas (DC, $\mathrm{mm}$ ) seguindo as recomendações da literatura ${ }^{(18)}$. Para a medida da MC foi utilizada uma balança da marca Plena com precisão de 100 gramas e a EST medida com um estadiômetro Cardiomed com precisão de $1 \mathrm{~mm}$. Em homens e mulheres foram mensuradas as DC subescapular e suprailíaca oblíqua, tricipital e panturrilha medial com um compasso tipo Cescorf com precisão de $0,1 \mathrm{~mm}$. Todos os instrumentos estavam devidamente calibrados.

A densidade corporal (D) foi estimada através das equações de Petroski(18), validadas para a população brasileira, tanto para homens (D $=1,10726863-0,00081201[\Sigma 4 D C]+0,00000212[\Sigma 4 D C]^{2}-0,00041761$ $[I D])$ quanto para mulheres $(D=1,02902361-0,00067159[\Sigma 4 D C]+$ $0,00000242[\Sigma 4 D C]^{2}-0,00026073[I D]-0,00056009[M C]+0,00054649$ [EST]) (ID: idade em anos). A estimativa do \% G foi calculada pela equação de Siri (\%G = [495/D] - 450)(18).

A determinação de riscos para saúde relacionados com sobrepeso/ obesidade foi realizada com base no \%G e IMC. Para a classificação através do \%G adotaram-se os critérios sugeridos por Heyward(119), que avalia os sujeitos em "com" (ㄱ': $\geq 25$ e 우: $\geq 32 \%$ ) e "sem risco". Para a classificação do IMC foram empregados os critérios sugeridos pela Organização Mundial de Saúde ${ }^{(20)}$, considerando excesso de peso valores $\geq 25 \mathrm{~kg} / \mathrm{m}^{2}$.

O inventário de razões para o exercício desenvolvido por Silberstein et al. ${ }^{(21)}$ é composto por 24 itens sobre motivos gerais para a prática de atividade física, divididos em sete categorias: controle de peso, aptidão física, saúde, melhora da harmonia do corpo, da atratividade física, da disposição e diversão. O avaliado deveria classificá-lo em uma escala de um a sete pontos de acordo com o grau de importância. A variável motivos para a prática do exercício foi obtida através dos escores atingidos pelo inventário. Os escores foram dados através da soma das respostas em cada categoria, sendo a importância do motivo determinada pelo maior ou menor escore.

A escala de avaliação da figura corporal proposta por Stunkard et al. e citada por Hart ${ }^{(4)}$ (figura 1), devidamente validada para a população brasileira(22), foi utilizada para estimar a satisfação com a imagem corporal a partir de um conjunto de figuras masculinas e femininas representando vários tamanhos corporais e numeradas de 1 a 9 . A escala apresenta correlações positivas significativas com os valores de \%G e $I M C^{(2)}$. O indivíduo deveria escolher a figura que mais se assemelha a ele (figura atual - FA), e ainda aquela que ele gostaria de apresentar (figura ideal - Fl). A satisfação com a imagem corporal foi determinada com o número da Fl subtraído do número da FA (FI - FA). A diferença fornece o grau de discrepância, o qual revela a insatisfação com a imagem corporal. A satisfação é um escore que quanto mais próximo de zero, indica maior grau de satisfação. Maiores escores indicam maior insatisfação com a imagem corporal.

Os indivíduos foram informados acerca dos procedimentos da pesquisa e assinaram o termo de consentimento informado seguindo as Diretrizes e Normas Regulamentadoras de Pesquisas Envolvendo Seres Humanos (Resolução no 196, de 10 de outubro de 1996) do Conselho Nacional de Saúde. O estudo foi aprovado pelo Comitê de Ética da PUCPR (processo no 1.135) e a pesquisa realizada entre os meses de agosto e setembro de 2006. 


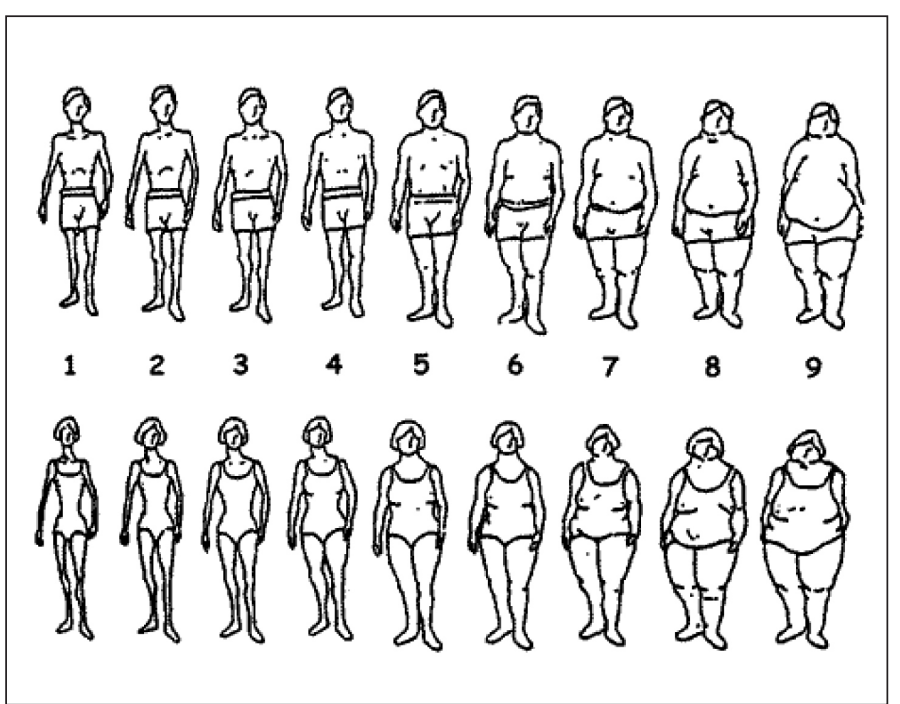

Figura 1. Escala de avaliação das figuras corporais (Stunkard et al.(4))

\section{Análise estatística}

A descrição dos escores de motivos para a prática de atividade física e imagem corporal foi realizada através da média e desvio-padrão, sendo os valores médios comparados entre os sexos, categorias de \%G e IMC através do teste $t$ de medidas independentes. A análise preliminar da associação entre imagem corporal, motivos para prática de atividade física, \%G e IMC foi realizada com a correlação linear de Pearson. As variáveis que apresentaram interação nas análises preliminares foram selecionadas para compor os modelos de regressão linear múltipla. A associação entre imagem corporal e os motivos para realizar atividade física foi analisada considerando sexo e IMC (modelo 1) e sexo e \%G (modelo 2) como variáveis controladoras, sendo a satisfação com a imagem corporal a variável independente em ambos os modelos. 0 nível de significância adotado foi de $5 \%$ e os dados foram analisados com o software SPSS 11.0.

\section{RESULTADOS}

Entre os participantes, 50\% eram homens; a maioria apresentava idade entre 21 e 30 anos (56,7\%), com proporções similares entre homens $(54,3 \%)$ e mulheres $(59,1 \%)$ nessa faixa etária. A escolaridade foi elevada: $45,6 \%$ dos indivíduos cursando ou com nível superior incompleto e $28,9 \%$ com grau completo (tabela 1). A prevalência de prática de atividade física no lazer foi elevada $(48,9 \%)$ em ambos os sexos (o’': 58,7 e 우: 38,6\%). Maior proporção de homens relatou utilizar o deslocamento ativo para o trabalho (39,1\%), realizar atividades de força $(32,6 \%)$ e de alongamento (41,3\%). Aproximadamente 31\% dos indivíduos apresentavam excesso de peso (o`: 41,3 e 우: 20,5\%) e 14,4\% possuíam \%G classificado como risco para a saúde (ð’: 17,4 e 우: 11,4\%).

Tabela 1. Distribuição dos participantes de acordo com características demográficas e de atividade física.

\begin{tabular}{c|c|c|c|c|c|c}
\hline & \multicolumn{2}{|c|}{$\begin{array}{c}\text { Homens } \\
(\mathrm{n}=45)\end{array}$} & \multicolumn{2}{c|}{$\begin{array}{c}\text { Mulheres } \\
(\mathrm{n}=45)\end{array}$} & \multicolumn{2}{c}{$\begin{array}{c}\text { Todos } \\
(\mathrm{n}=90)\end{array}$} \\
\hline & $\mathrm{n}$ & $\%$ & $\mathrm{n}$ & $\%$ & $\mathrm{n}$ & $\%$ \\
\hline
\end{tabular}

\section{Faixa etária (anos)}

\begin{tabular}{l|c|c|c|c|c|c}
\hline$\leq 20$ & 9 & 19,6 & 6 & 13,6 & 15 & 16,7 \\
\hline $21-30$ & 25 & 54,3 & 26 & 59,1 & 51 & 56,7 \\
\hline $31-40$ & 5 & 10,9 & 7 & 15,9 & 12 & 13,3 \\
\hline$>40$ & 7 & 15,2 & 5 & 11,4 & 12 & 13,3 \\
\hline
\end{tabular}

\section{Escolaridade}

\begin{tabular}{l|c|c|c|c|c|c}
\hline Ensino médio incompleto & 7 & 15,2 & 3 & 6,8 & 10 & 11,1 \\
\hline Ensino médio completo & 7 & 15,2 & 6 & 13,6 & 13 & 14,4 \\
\hline Ensino superior incompleto & 17 & 37 & 24 & 54,6 & 41 & 45,6 \\
\hline Ensino superior completo & 15 & 32,6 & 11 & 25 & 26 & 28,9 \\
\hline
\end{tabular}

\section{Atividade física ( $\geq 3 \times$ semana)}

\begin{tabular}{l|c|c|c|c|c|c}
\hline Deslocamento ao trabalho & 18 & 39,1 & 9 & 20,5 & 27 & 30 \\
\hline Atividade física no lazer & 27 & 58,7 & 17 & 38,6 & 44 & 48,9 \\
\hline Atividades de força & 15 & 32,6 & 4 & 9,1 & 19 & 21,1 \\
\hline Atividades de alongamento & 19 & 41,3 & 12 & 27,3 & 31 & 34,4 \\
\hline
\end{tabular}

A tabela 2 apresenta os escores dos motivos para a prática de atividade física em ordem decrescente e a insatisfação com a imagem corporal. Independente do sexo, \%G e IMC, os maiores escores foram encontrados nas questões saúde, aptidão física, disposição, atratividade e harmonia. Na comparação entre os sexos, entre as mulheres o motivo harmonia apresentou diferença significativa $(p=0,03)$. Esse fato também foi verificado entre os indivíduos "com risco" de acordo com o \%G. Contudo, o mesmo não ocorreu quando o IMC foi empregado como critério. Em relação à insatisfação com a imagem corporal, as mulheres apresentaram maior escore, assim como os indivíduos "com risco" (\%G) e com "excesso de peso" (IMC). É importante ter em mente que essa variável é uma medida de insatisfação corporal e que, quanto maior o índice, maior a insatisfação.

Tabela 2. Escores dos motivos para a prática de atividade física e de insatisfação com a imagem corporal de acordo com sexo, \%G e IMC.

\begin{tabular}{|c|c|c|c|c|c|c|}
\hline \multirow[b]{2}{*}{ Motivos } & \multicolumn{2}{|c|}{ Sexo } & \multicolumn{2}{|c|}{$\% G$} & \multicolumn{2}{|c|}{ IMC } \\
\hline & Homens & Mulheres & Com risco & Sem risco & Excesso de peso & Normal \\
\hline Saúde & $23,2 \pm 4,5$ & $23,8 \pm 3,9$ & $24,4 \pm 2,9$ & $23,3 \pm 4,4$ & $23,1 \pm 3,5$ & $23,6 \pm 4,5$ \\
\hline Aptidão física & $22,4 \pm 3,8$ & $22 \pm 3,6$ & $21,9 \pm 3,3$ & $22,2 \pm 3,7$ & $20,8 \pm 3,5$ & $22,9 \pm 3,6$ \\
\hline Disposição & $18,3 \pm 5,9$ & $18,7 \pm 6$ & $19,8 \pm 5,6$ & $18,3 \pm 5,9$ & $18,2 \pm 6,1$ & $18,6 \pm 5,8$ \\
\hline Atratividade & $14 \pm 4,6$ & $15,2 \pm 3,8$ & $16 \pm 4,4$ & $14,4 \pm 4,2$ & $14,6 \pm 4,5$ & $14,6 \pm 4,2$ \\
\hline Harmonia & $13,9 \pm 3,9$ & $15,7 \pm 3,9^{*}$ & $16,5 \pm 1,7^{*}$ & $14,5 \pm 4,2$ & $15 \pm 3,3$ & $14,6 \pm 4,2$ \\
\hline Diversão & $11,6 \pm 4,2$ & $10 \pm 4,6$ & $11,2 \pm 4$ & $10,7 \pm 4,5$ & $10,8 \pm 4,2$ & $10,8 \pm 4,5$ \\
\hline Controle de peso & $8,4 \pm 3,2$ & $9,5 \pm 2,5$ & $10,5 \pm 2,1$ & $8,6 \pm 3$ & $10 \pm 2,3$ & $8,5 \pm 3,1$ \\
\hline Insatisfação com a imagem corporal & $0,4 \pm 1,5$ & $1,0 \pm 1,2^{*}$ & $2,1 \pm 1,3^{*}$ & $0,4 \pm 1,3$ & $1,6 \pm 1,4^{*}$ & $0,3 \pm 1,2$ \\
\hline
\end{tabular}

valores expressos em média e desvio padrão, ${ }^{*} p<0,05$ 
A análise bivariada (tabela 3) demonstrou que o motivo controle de peso está associado de maneira positiva com o \% $(r=0,46 ; p<0,001)$, IMC $(r=0,33 ; p<0,001)$ e insatisfação com a imagem corporal $(r=0,26$; $p<0,01)$. Entre os outros motivos analisados, a harmonia apresentou-se correlacionada de maneira positiva com o \%G $(r=0,34 ; p<0,001)$ e a insatisfação com a imagem corporal $(r=0,32 ; p<0,001)$.

Tabela 3. Valores de correlação de Pearson entre os escores dos motivos para prática de atividade física, \%G, IMC e insatisfação com a imagem corporal.

\begin{tabular}{l|c|c|c}
\hline & \%G & IMC & $\begin{array}{c}\text { Insatisfação com a } \\
\text { imagem corporal }\end{array}$ \\
\hline Controle de peso & $0,456^{* *}$ & $0,329^{* *}$ & $0,26^{*}$ \\
\hline Harmonia & $0,341^{* *}$ & 0,130 & $0,318^{* *}$ \\
\hline Aptidão física & -0179 & $-0,203$ & $-0,133$ \\
\hline Atratividade & 0,078 & 0,129 & 0,090 \\
\hline Diversão & $-0,129$ & 0,055 & $-0,002$ \\
\hline Disposição & 0,099 & 0,026 & $-0,016$ \\
\hline Saúde & 0,068 & 0,018 & 0,049 \\
\hline *0<0,01***< & & &
\end{tabular}

${ }^{*} p<0,01 ;{ }^{* *} p<0,001$

Os motivos controle de peso e harmonia foram analisados separadamente como variáveis dependentes, tendo como variável independente a satisfação com a imagem corporal (tabela 4). Ambos apresentaram associação com os dois modelos de análise empregados (controle de peso: $F=6,09 ; p=0,001$ e $F=8,26 ; p=0,000$; harmonia: $F=4,14 ; p=0,009$ e $F=4,59 ; p=0,005)$. No entanto, a análise das correlações parciais demonstrou que o controle de peso não estava associado à satisfação corporal após ajuste para as variáveis controladoras em ambos os modelos (modelo $1: \beta=-0,03$ e $p=0,81$; modelo $2: \beta=-0,07$ e $p=0,55$ ). A variável harmonia, após ajuste para as variáveis controladoras, apresentou associação apenas no modelo 1 $(\beta=0,28 ; p=0,03)$.

Tabela 4. Valores dos modelos de análise de regressão linear entre os motivos controle de peso e harmonia e satisfação com a imagem corporal.

\begin{tabular}{l|c|c|c|c|c|c|c|c|c|c}
\hline \multirow{2}{*}{$\begin{array}{l}\text { Variáveis } \\
\text { dependentes }\end{array}$} & \multicolumn{5}{c|}{$\begin{array}{c}\text { Modelo 1 } \\
\text { Sexo e IMC }\end{array}$} & \multicolumn{5}{c}{$\begin{array}{c}\text { Modelo 2 } \\
\text { Sexo e \%G }\end{array}$} \\
\cline { 2 - 11 } & $R^{2}$ aju & $F$ & $p$ & $\beta$ & $p$ & $R^{2}$ aju & $F$ & $p$ & $\beta$ & $p$ \\
\hline $\begin{array}{l}\text { Controle de } \\
\text { peso }\end{array}$ & 0,14 & 6,09 & 0,001 & $-0,03$ & 0,81 & 0,19 & 8,26 & 0,000 & $-0,07$ & 0,55 \\
\hline Harmonia & 0,09 & 4,14 & 0,009 & 0,28 & 0,03 & 0,10 & 4,59 & 0,005 & 0,19 & 0,14 \\
\hline
\end{tabular}

\section{DISCUSSÃO}

Este estudo exploratório buscou identificar os motivos para a prática de atividade física e analisar sua relação com a imagem corporal em indivíduos adultos frequentadores de academia, considerando o $\%$ G e IMC. Nesse sentido, o estudo apresenta contribuições originais, uma vez que são escassas pesquisas similares na literatura.

A amostra não é representativa dos indivíduos que praticam atividade física em academias da cidade de Curitiba-PR, sendo composta por homens e mulheres voluntários; contudo, a seleção dos sujeitos ocorreu entre os que praticavam as atividades regularmente.

Aproximadamente $75 \%$ dos sujeitos apresentavam elevado grau de escolaridade. Essa é uma característica esperada, uma vez que entre indivíduos com maior escolaridade é verificada prevalência superior de atividade física no lazer ${ }^{(12,23)}$. Existem evidências de que sujeitos com nível superior completo apresentam cerca de oito vezes mais chance de praticar exercícios comparados com aqueles com ensino fundamental incompleto ${ }^{(14)}$. Cerca de 49\% dos indivíduos relataram praticar atividade física no lazer. Esse valor é superior à média populacional da cidade, onde apenas 14\% relataram tal prática ${ }^{(12)}$. Essa diferença pode ser atribuída ao critério de seleção da amostra e classificação de atividade física de lazer. Em relação ao IMC, 31\% dos indivíduos apresentaram excesso de peso $\left(\geq 25 \mathrm{~kg} / \mathrm{m}^{2}\right)$, sendo o valor inferior aos $44,9 \%$ verificados na população de Curitiba-PR ${ }^{(12)}$

Os motivos para a prática de atividade física que apresentaram maiores escores foram saúde, aptidão física, disposição, atratividade e harmonia. No estudo realizado por Tahara et al.(10) com 50 indivíduos jovens ( $\leq 24$ anos), os motivos mais citados foram as questões estéticas, melhoria da qualidade de vida e aptidão física. O nível de escolaridade era diversificado, o que pode, em parte, explicar as diferenças encontradas, uma vez que indivíduos com maior escolaridade apresentam percepções e conhecimentos diferenciados em relação à prática de exercícios e seus benefícios. Balbinotti e Capozzoli(16) encontraram resultados que corroboram, em parte, os verificados neste estudo. Para os indivíduos de 21 a 40 anos, os motivos mais importantes para a prática de atividade física foram saúde, prazer, controle do estresse e estética. Os autores verificaram ainda que o motivo saúde apresentou maior número de relatos com o incremento da idade, enquanto a razão competitividade diminuiu ${ }^{(16)}$. Na pesquisa de Araújo e Araújo(17), 85\% dos sujeitos relataram procurar a prática de exercícios com o objetivo de melhora da qualidade de vida ou prevenção/tratamento de doenças, enquanto 10\% reportaram motivos como estética, prazer e lazer. É importante salientar que a idade dos indivíduos era de 53,2 \pm 15,2 anos (22-85 anos) e que 89\% deles apresentavam condição clínica de doença cardiovascular ou outras doenças, o que possivelmente tenha influenciado nos objetivos. De Andrade Bastos et al. ${ }^{(15)}$ verificaram que os homens e os adultos jovens são mais motivados por questões sociais, enquanto pessoas com maior grau de instrução, por causa da saúde e aptidão física. Shen e $\mathrm{Xu}^{(9)}$ encontraram resultados semelhantes em universitários chineses. Os resultados apontam para grande diversidade de razões para a exercício em função do sexo, faixa etária, grau de instrução, estado de saúde, instrumento utilizado e modo como os indivíduos foram questionados.

No presente estudo, o escore do motivo harmonia foi significativamente maior entre as mulheres, o que parece confirmar o fato de ideias diferenciadas das percepções em relação aos homens ${ }^{(4,9)}$. Foi observado que indivíduos com \%G elevado apresentaram maior escore do motivo harmonia. Porém, quando o IMC foi utilizado como indicador, não houve diferença entre os indivíduos que apresentam peso normal e excesso de peso. Em parte, esse resultado pode ser atribuído às limitações em relação ao uso do IMC, o qual não permite diferenciar a composição corporal(24). Essa limitação poder ser potencializada pelo tipo de atividade praticada em academia, como a musculação, a qual pode levar os praticantes a desenvolver maior quantidade de massa muscular. Foi observado que as mulheres e os indivíduos com maiores valores de \%G e IMC apresentaram maior insatisfação com a imagem corporal, sugerindo que esta é influenciada pelo sexo e os riscos relacionados com sobrepeso/obesidade. Araújo e Araújo ${ }^{(24)}$ já haviam verificado que especialmente as mulheres são insatisfeitas com sua massa corporal e gostariam de pesar, em média, 3,3kg menos. Entretanto, Damasceno et al. ${ }^{(2)}$ não verificaram diferenças no grau de insatisfação com a imagem corporal 
entre os sexos. Em relação ao IMC também existem evidências de que indivíduos com sobrepeso/obesidade apresentam imagens corporais negativas ${ }^{(4,8)}$. Millstein et al. ${ }^{(8)}$, por exemplo, verificaram que a prevalência de insatisfação com o tamanho corporal é maior entre as mulheres com excesso de peso.

Existem consistentes evidências de que o exercício proporciona melhoria no bem-estar psicológico, autoestima e imagem corporal $^{(11)}$. Hart( ${ }^{(4)}$ afirma que esse é um objetivo comum entre os indivíduos que iniciam programas de exercícios. Teixeira et al. ${ }^{(5)}$ verificaram que a motivação para o exercício esteve significativamente relacionada de maneira positiva com maior autoeficácia, conceito físico e atratividade corporal, e negativamente associada à percepção de barreiras. Apesar dos benefícios supracitados, diversas razões para não praticar atividade física foram apontadas na literatura ${ }^{(25,26)}$ Em um estudo de base populacional com adultos de Pelotas-RS, Reichert et al. ${ }^{(26)}$ encontraram entre os indivíduos jovens (20-49 anos) a falta de dinheiro, a sensação de cansaço, a falta de companhia e a falta de tempo como as principais barreiras. Verificou-se tendência de linearidade entre o número de barreiras relatadas e a prevalência de inatividade física. Os indivíduos que não praticavam atividade física por falta de tempo apresentaram 36\% mais chance de ser inativos. Em se tratando de programas de exercícios, a identificação das possíveis barreiras para a sua prática representa uma importante ferramenta de subsídio para organização e promoção da atividade física de maneira eficaz, fato que pode potencializar a aderência ao programa(25).

Maiores valores de \%G, IMC e insatisfação com a imagem corporal estiveram relacionados com o controle de peso e harmonia (exceto IMC) como motivo para a prática de atividade física. A isso pode ser atribuído que grande parte dos indivíduos insatisfeitos com sua aparência física e a harmonia corporal buscam programas de exercícios para controle/redução da massa corporal, melhora da estética e/ou manutenção da forma física ${ }^{(2,9)}$. O caso da harmonia apresentar-se relacionada somente com o \%G pode sugerir que o fator de influência desse motivo é a composição corporal, e não a massa corporal total. O motivo aptidão física apresentou correlação negativa, porém não significativa, com o IMC, \%G e insatisfação com a imagem corporal, sugerindo possível relação inversa. Esse fato já tinha sido observado por Adame et al.(27) em seu estudo, no qual maiores níveis de aptidão foram associados a boa imagem corporal.

Embora a insatisfação com a imagem corporal não tenha apresentado associação com o motivo controle de peso na análise multivariada, existe maior efeito observado no modelo que empregou o $\% \mathrm{G}(\mathrm{F}=8,26)$ do que naquele com o IMC ( $F=6,09)$, sugerindo que a quantidade de gordura corporal (\%G) possa exercer maior influência no motivo de perda/controle de peso do que a massa corporal total do indivíduo (IMC). Esse fato poderia ser justificado pelas limitações e críticas ao uso do IMC ${ }^{(24)}$. Como ele não discrimina os efeitos do excesso de peso provenientes de massa muscular e gordura, indivíduos com valores semelhantes podem ter diferentes representações clínicas. Por exemplo, valores elevados de IMC podem ser consequência apenas de um intenso programa de exercícios de fortalecimento muscular em indivíduos com baixos níveis de gordura.

O motivo harmonia apresentou associação com a insatisfação corporal; no entanto, quando a influência da insatisfação foi analisada independente do sexo e índices de obesidade (\%G e IMC), somente foi observada associação quando a variável controladora foi o IMC. Essas constatações sugerem que a influência da imagem corporal nesse motivo não está relacionada com a composição corporal e sim com a massa corporal total. Pode-se inferir que a quantidade de gordura não é associada a esse motivo, mas sim, uma questão mais introspectiva, de como o indivíduo se sente em relação ao próprio corpo ${ }^{(4)}$. De acordo com Hart ${ }^{(4)}$, a harmonia é um fator relacionado com a imagem corporal. Existem evidências de que a maioria dos indivíduos que iniciam a prática de atividade física busca modificar suas formas e proporções corporais motivados pela insatisfação com a imagem corporal ${ }^{(1,2,4)}$. Os homens procuram um corpo mais forte, volumoso e com baixo $\% \mathrm{G}^{(2,3,7,28)}$, enquanto as mulheres preferem um corpo mais magro e menos volumoso ${ }^{(2,3,6)}$. Devido à dificuldade em conseguir alcançar tais dimensões, a maior parte dos indivíduos apresenta insatisfação com sua imagem corporal independente do sexo ${ }^{(2,3,11)}$. Millstein et al. ${ }^{(8)}$ verificaram que a insatisfação com o tamanho corporal é maior entre as mulheres (23,6\% versus $12,6 \%)$. No estudo de Damasceno et al. ${ }^{(2)}$, $82 \%$ dos homens e $76 \%$ das mulheres estavam insatisfeitos com sua imagem corporal.

Os pesquisadores e profissionais da atividade física têm concentrado suas atenções para medidas corporais objetivas, deixando em segundo plano a avaliação subjetiva das percepções corporais ${ }^{(4)}$. Damasceno et al.(2) utilizaram a mesma escala de percepção da figura corporal para avaliar adultos jovens (28,7 $\pm 12,6$ anos) e observaram correlações positivas significativas entre o escore da escala com o \%G

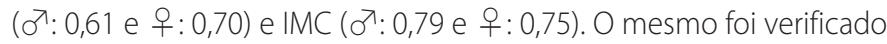
por Scagliusi et al. ${ }^{(22)}$ em relação ao IMC.

Embora o presente estudo tenha contribuído para melhor compreensão acerca da relação entre os motivos para a prática de atividade física e a insatisfação com a imagem corporal, algumas limitações devem ser consideradas para a interpretação dos resultados. Além do viés de seleção da amostra anteriormente referenciado e a limitação acerca da utilização da escala de avaliação da figura corporal já apontada na literatura ${ }^{(2,22)}$, não é possível determinar a causalidade da relação entre as variáveis, uma vez que se trata de um estudo de corte transversal. Os participantes da pesquisa eram frequentadores de academia e, portanto, com maior motivação para a prática de atividade física, o que pode provocar um efeito de "teto" nos escores utilizados. Por essa razão, outras pesquisas com inclusão de amostras mais heterogêneas e representativas devem ser conduzidos para verificar a consistência dos resultados.

\section{CONCLUSÕES}

Com base nos resultados, pode-se concluir que 1) os motivos de maior importância para a prática de atividade física foram saúde, aptidão física, disposição, atratividade e harmonia; 2) mulheres e indivíduos com elevado \%G atribuem a prática de atividade física ao motivo harmonia, assim como aqueles insatisfeitos com a imagem corporal buscam as atividades para o controle de peso e harmonia; 3) mulheres e indivíduos com maior \%G e IMC apresentam maior insatisfação com a imagem corporal; 4) a insatisfação com a imagem corporal parece estar mais associada a aspectos relacionados com o bem-estar do que com a condição física.

Os achados deste estudo têm implicações para os programas estruturados de atividade física, tais como aqueles oferecidos em academias, clubes e clínicas. Esses devem considerar o bem-estar e a qualidade de vida como aspectos motivacionais importantes entre os frequentadores, ainda que a imagem corporal possa não ser um fator decisivo para a motivação de sujeitos com riscos relacionados com sobrepeso/ obesidade na prática de atividade física.

Todos os autores declararam não haver qualquer potencial conflito de interesses referente a este artigo. 


\section{REFERÊNCIAS BIBLIOGRÁFICAS}

1. Damasceno VO, Vianna VRA, Vianna JM, Lacio M, Lima JRP, Novaes JS. Imagem corporal e corpo ideal. Rev Bras Ci e Mov 2006;14:81-94.

2. Damasceno VO, Lima JRP, Vianna JM, Vianna VRA, Novaes JS. Tipo físico ideal e satisfação com a imagem corporal de praticantes de caminhada. Rev Bras Med Esporte 2005;11:181-6.

3. Kakeshita IS, Almeida SS. Relação entre índice de massa corporal e a percepção da auto-imagem em universitários. Rev Saúde Pública 2006;40:497-504.

4. Hart EA. Assessing body image. In: Tritschler KA. Practical measurement and assessment. Baltimore: Lippincott Williams \& Wilkins; 2000. p. 409-37.

5. Teixeira PJ, Going SB, Houtkooper LB, Cussler EC, Metcalfe LL, Blew RM, et al. Exercise motivation, eating and body image variables as predictors of weight control. Med Sci Sports Exerc 2006;38:179-88.

6. Pedretti A. Imagem corporal e morfologia: estudo comparativo entre mulheres brasileiras e portuguesas praticantes de musculação (Dissertação de Mestrado - Programa de Pós-Graduação em Ciências do Desporto). Porto: Universidade do Porto; 2008

7. Blond A. Impacts of exposure to images of ideal bodies on male body dissatisfaction: a review. Body Image 2008:5:244-50.

8. Millstein RA, Carlson SA, Fulton JE, Galuska DA, Zhang J, Blanck HM, et al. Relationships between body size satisfaction and weight control practices among US adults. Medscape J Med 2008;10:119.

9. Shen B, Xu C. Effects of self-efficacy, body mass, and cardiorespiratory fitness on exercise motives in chinese college students. J Phys Activ Health 2008;5:706-18.

10. Tahara AK, Schwartz GM, Silva KA. Aderência e manutenção da prática de exercícios em academias. Rev Bras Ci e Mov 2003;11:7-11.

11. Hausenblas HA, Fallon EA. Exercise and body image: a meta-analysis. Psychol Health 2006;21:33-47.

12. Vigitel Brasil 2007. Vigilância de fatores de risco e proteção para doenças crônicas por inquérito telefônico. http://bvsms.saude.gov.br (Acessado em 20/dez/08).

13. Malta DC, Moura EC, Castro AM, Cruz DKA, Neto OLM, Monteiro CA. Padrão de atividade física em adultos brasileiros: resultados de um inquérito por entrevistas telefônicas, 2006. Epidemiol Serv Saúde 2009;18:7-16

14. Florindo $A A$, Latorre MRDO, Tanaka T, Jaime PC, Zerbini CAF. Fatores associados à prática de exercícios físicos em homens voluntários adultos e idosos residentes na Grande São Paulo, Brasil. Rev Bras Epidemiol 2001;4:105-13.
15. de Andrade Bastos A, Salguero A, González-Boto R, Marquez S. Motives for participation in physical activity by Brazilian adults. Percept Mot Skills 2006;102:358-67.

16. Balbinotti MAA, Capozzoli CJ. Motivação à prática regular de atividade física: estudo exploratório com praticantes em academias de ginástica. Rev Bras Educ Fís Esp 2008;22:63-80

17. Araújo DSMS, Araújo CGS. Autopercepção corporal de variáveis da aptidão física relacionada à saúde Rev Bras Med Esporte 2002;8:37-49.

18. Petroski EL. Antropometria: Técnicas e padronizações. Porto Alegre; 2003.

19. Heyward VH. Avaliação da composição corporal aplicada. São Paulo; Manole; 2000

20. WHO. Physical status: the use and interpretation of anthropometry. Report of a WHO Expert Committee (1995) (Technical Report Series, № 854). http://www.who.int/childgrowth/publications/ physical_status/en/index.html (Acessado em 15/out/06).

21. Silberstein LR, Striegel-Moore RH, Timko C, Rodin J. Behavioral and psychological implications of body dissatisfaction: Do men and women differ? Sex Roles 1988;19:219-32.

22. Scagliusi FB, Alvarenga M, Polacow VO, Cordas TA, de Oliveira Queiroz GK, Coelho D, et al. Concurrent and discriminant validity of the Stunkard's figure rating scale adapted into Portuguese. Appetite 2006; $47: 77-82$

23. Hallal PC, Reichert FF, Siqueira FV, Dumith SC, Bastos JP, Silva MC, et al. Correlates of leisure-time physica activity differ by body-mass-index status in brazilian adults. J Phys Activ Health 2008;5:571-8.

24. Araújo DSMS, Araújo CGS. Autopercepção e insatisfação com peso corporal independem da freqüência de atividade física. Arq Bras Cardiol 2003;80:235-42.

25. Seefeldt V, Malina RM, Clark MA. Factors affecting levels of physical activity in adults. Sports Med 2002;32:143-68.

26. Reichert FF, Barros AJ, Domingues MR, Hallal PC. The role of perceived personal barriers to engagement in leisure-time physical activity. Am J Public Health 2007;97:515-9.

27. Adame DD, Johnson TC, Cole SP, Matthiasson H, Abbas MA. Physical fitness in relation to amount of physical exercise, body image, and locus of control among college men and women. Percept Mot Skills 1990;70:1347-50.

28. Halliwell $\mathrm{E}$, Dittmar $\mathrm{H}$, Orsborn $\mathrm{A}$. The effects of exposure to muscular male models among men: exploring the moderating role of gym use and exercise motivation. Body Image 2007:4:278-87. 